\title{
Aberrant allelic-switch of antisense IncRNA IRAIN may be an early diagnostic marker in laryngeal cancer
}

\author{
JIAN YAN WANG ${ }^{1,2}$, DANQING LIU ${ }^{3}$, YING DI MENG ${ }^{1}$, YING YUAN GUO ${ }^{1}$ and MING ZHAO ${ }^{1}$ \\ ${ }^{1}$ Department of Otorhinolaryngology Head and Neck Surgery, Second Hospital of Jilin University, Changchun, Jilin 130041; \\ ${ }^{2}$ Department of Otorhinolaryngology Head and Neck Surgery, Third Affiliated Hospital of \\ Sun Yat-Sen University, Guangzhou, Guangdong 510000; ${ }^{3}$ Department of Otorhinolaryngology Head and Neck Surgery, \\ First Affiliated Hospital of Sun Yat-sen University, Guangzhou, Guangdong 510080, P.R. China
}

Received December 3, 2019; Accepted June 29, 2020

DOI: $10.3892 / \mathrm{ol} .2020 .11926$

\begin{abstract}
Laryngeal carcinoma is a common head and neck malignancy, however, the molecular mechanism of the disease has not yet been elucidated. The present study aimed to investigate the role of IGF1R antisense imprinted non-protein coding RNA (IRAIN) long non-coding (lnc) RNA in laryngeal carcinoma. In total, specimens of healthy pharynx tissue from 6 healthy individuals, carcinoma tissue and paracancerous tissue from 37 patients with laryngeal carcinoma were used in this study. The single nucleotide polymorphism (SNP) rs8034564 was used to distinguish the two parental alleles of IRAIN. DNA and RNA were extracted from tissue specimens and the IRAIN allelic gene was sequenced. Reverse transcription-quantitative PCR was used to determine the expression levels of IRAIN and Insulin-like growth factor 1 receptor (IGF1R) in laryngeal carcinoma and paracancerous tissue. Bisulfite genomic sequencing was used to determine IRAIN promoter DNA methylation status in laryngeal carcinoma tissue. The expression of IRAIN was di-allelic in healthy pharynx tissue, laryngeal carcinoma tissue and paracancerous tissue. Moreover, IRAIN expression in laryngeal carcinoma tissue was lower compared with paracancerous tissue $(\mathrm{P}<0.05)$. IRAIN expression was not associated with age, histological type, tumor stage and grade and lymph node metastasis. IRAIN allelic expression imbalance was present in laryngeal carcinoma and paracancerous tissue, but not in healthy pharynx tissue. SNP analysis (rs8034564) indicated there was an allelic-switch of the two parental alleles. Furthermore, epigenetic analysis revealed no extensive DNA
\end{abstract}

Correspondence to: Dr Ming Zhao, Department of Otorhinolaryngology Head and Neck Surgery, Second Hospital of Jilin University, 218 Ziqiang, Nanguan, Changchun, Jilin 130041, P.R. China

E-mail: mzhao89@yahoo.com

Key words: laryngeal carcinoma, long non-coding RNA IGF1R antisense imprinted non-protein coding RNA, allelic-switch, epigenetics, genomic imprinting methylation of $\mathrm{CpG}$ islands in the IRAIN gene promoter of laryngeal carcinoma. Therefore, it was suggested that IRAIN allele was non-imprinted in laryngeal carcinoma and healthy pharynx tissue. It was also demonstrated that IRAIN may be a potential tumor suppressor in laryngeal carcinoma, and that DNA methylation is not involved in the regulation of IRAIN gene immobilization in laryngeal carcinoma tissue. Thus, detection of IRAIN allelic expression imbalance and aberrant allele-switch may serve as an early diagnostic marker of laryngeal carcinoma.

\section{Introduction}

Laryngeal carcinoma is a common head and neck malignancy, and squamous cell carcinoma is the most frequent histological type of laryngeal carcinoma, accounting for $98 \%$ of cases $(1,2)$. The main risk factors for laryngeal carcinoma are tobacco smoking and alcohol consumption, and the roles of these risk factors have been reported by a large number of studies $(3,4)$. While much is known regarding laryngeal carcinoma, the molecular mechanism of the disease is yet to be elucidated.

Insulin-like growth factor 1 (IGF-1) signaling is mediated via the IGFR1 receptor (IGF1R), which is an important growth regulatory pathway, and the enhanced activation of this pathway is indicated to serve a crucial role in cancer cell proliferation, migration and apoptosis (5-7). Furthermore, IGF1R is aberrantly expressed in numerous types of cancer cells, including laryngeal carcinoma (8).

IGF1R antisense imprinted non-protein coding RNA (IRAIN) is a newly reported antisense imprinted non-coding RNA in the IGF1R locus, which has an important role in the regulation of IGF-1 signaling (9). IRAIN is transcribed from an intronic promoter with antisense orientation and with a parent-of-origin genetic manner $(9,10)$. The present study examined a single nucleotide polymorphism (SNP) of IRAIN (rs8034564), and revealed the 'A' genotype was favorably imprinted over the ' $G$ ' genotype, resulting in imbalanced expression in the two parental alleles (10). IRAIN has been shown to be downregulated in leukemia cell lines and fresh blood cells of patients with high-risk acute myeloid leukemia (AML) (9), and is downregulated in breast cancer as an imprinted gene (10). Another previous study reported 
that IRAIN was significantly increased in non-small cell lung carcinoma (NSCLC) tissues, was related to tumor size and smoking status and that downregulation of IRAIN suppressed the proliferation of NSCLC cells via blocking cells in $\mathrm{G}_{1}$ phase (11). Pashaiefar et al demonstrated, in 64 de novo non-M3 patients with AML compared with 51 healthy controls that poor prognosis with low IRAIN expression tended to have a higher white blood cell count and blast count, and had markedly shorter overall survival (OS) and relapse-free survival (RFS; $\mathrm{P}=0.044$ and 0.009 , respectively); in addition, the study found that patients with a refractory response to chemotherapy and those with subsequent relapse, had lower initial IRAIN expression and multivariate analysis identified IRAIN transcript expression as an independent prognostic factor for OS and RFS (12). Previous studies have reported that the IGF1R pathway is frequently dysregulated in laryngeal carcinoma $(8,13)$; however, it has not been determined whether IRAIN serves a role in laryngeal carcinoma. Therefore, the aim of the present study was to investigate whether IRAIN was differently expressed in laryngeal carcinoma tissue, paracancerous tissue and healthy pharynx tissue.

\section{Materials and methods}

Patients. Patients with laryngeal carcinoma treated at The Second Affiliated Hospital of Jilin University between August 2014 and August 2017 were recruited for this study. The study was approved by the Research Ethics Committee of The Second Affiliated Hospital of Jilin University, and written informed consent was obtained from all patients.

Laryngeal carcinoma tissue and adjacent non-malignant tissue (distance from the malignant tissue, $0.5-1.5 \mathrm{~cm}$ ) was collected from 37 patients who received laryngectomy (some tissues were too small to cover all the assays, thus the sample number varied in each assay). The patient cohort consisted of 33 males and 4 females with an age range of 43-79 years old and a mean age of 60.2 years. In addition, healthy pharynx tissue was collected from 6 healthy individuals who received uvulopalatopharynoplasty. All tissue specimens were collected before patients had any radiotherapy and chemotherapy. After excision, all tissue samples were immediately stored at $-80^{\circ} \mathrm{C}$ until use.

Histological type and TNM classifications were determined according to the National Comprehensive Cancer Network (NCCN) criteria (14). All malignant tissue samples were examined by a pathologist, and determined to be epithelial squamous cell carcinoma. The clinical and pathological characteristics of the patients are presented in Table I.

\section{Evaluation of genomic imprinting}

Genomic DNA (gDNA) PCR product gene sequencing. gDNA was extracted using the Animal Tissue Genomic DNA Extraction kit (Beijing Zoman Biotechnology Co., Ltd.), following the manufacturer's instructions. Primers were designed according to the known SNP sites (10) (rs8034564; A/G) in the IRAIN expression region reported in the National Center for Biotechnology Information $(9,10)$. The IRAIN forward (JH248) and reverse (JH781) sequences (Table II) were selected for target fragment amplification. Target fragments were obtained using 2X Taq PCR MasterMix (Tiangen Biotech Co., Ltd.), according
Table I. Basic characteristics and pathological features of patients $(n=37)$ with laryngeal carcinoma involved in the experiment.

\begin{tabular}{lc}
\hline Characteristic & Patients \\
\hline Age, years & $61(43-79)$ \\
Sex & \\
Male & $33(89.2 \%)$ \\
Female & $4(10.8 \%)$ \\
TNM stage & \\
T1-T2 stage & $15(40.5 \%)$ \\
T3-T4 stage & $22(59.5 \%)$ \\
Histological type & \\
Highly differentiation & $12(32.4 \%)$ \\
Medium and low differentiation & $25(67.6 \%)$ \\
Lymph nodes metastasis & $11(29.7 \%)$ \\
Yes & $26(70.3 \%)$ \\
No & \\
Clinical classification & $19(51.4 \%)$ \\
Glottic carcinoma & $18(48.6 \%)$ \\
Supraglottic carcinoma &
\end{tabular}

Data are presented as the median and range or $\mathrm{n}(\%)$.

to the manufacturer's instructions. PCR reaction condition was as follows: Initial denaturation at $94^{\circ} \mathrm{C}$ for $3 \mathrm{~min}$, then IRAIN DNA was amplified for 30 cycles at $94^{\circ} \mathrm{C}$ for $30 \mathrm{sec}, 55^{\circ} \mathrm{C}$ for $30 \mathrm{sec}$ of annealing and $72^{\circ} \mathrm{C}$ for $1 \mathrm{~min}$ of extension, with a final extension at $72^{\circ} \mathrm{C}$ for $5 \mathrm{~min}$. PCR products $(5 \mu \mathrm{l} /$ well $)$ from each sample in sequence underwent electrophoresis for $20 \mathrm{~min}$ at $120 \mathrm{~V}$ on $2 \%$ agarose gel in an electrophoresis tank containing 1X TAE electrophoresis solution (Beijing Zoman Biotechnology Co., Ltd.); ethidium bromide was used to image the agarose gels. After electrophoresis, the gels were observed under a Tanon-4200 Gel Imaging System (Tanon Science and Technology Co., Ltd.). A bright electrophoresis band with clear edges was visible at $\sim 450 \mathrm{bp}$, and the target strip was excised from the gel and purified using a SGMag Gel DNA Purification kit (Sangon Biotech Co., Ltd.), and sent to the Shanghai Sangon Biotech Co., Ltd. for gene sequencing. Complementary DNA (cDNA) PCR product gene sequencing was performed if the SNP site in the sequencing results was $\mathrm{A} / \mathrm{G}$.

cDNA PCR product gene sequencing. Total RNA was extracted from frozen tissue samples using an AxyPrep Total RNA Extraction kit (Corning, Inc.) following the manufacturer's protocol. For reverse transcription (RT), $500 \mathrm{ng}$ total RNA was converted to cDNA using the Universal RT-PCR kit (Beijing Dingguo Changsheng Biotechnology Co., Ltd.) according to the manufacturer's protocol. Allelic expression of IRAIN was examined via PCR (Universal RT-PCR kit from Beijing Dingguo Changsheng Biotechnology Co., Ltd., the DNA polymerase was part of this kit.) in cDNA samples using primers specific for polymorphic restriction enzymes; the target fragments IRAIN JH780 and JH781 were selected for amplification (Table II). 
Table II. List of all primers.

A, Examination of genomic imprinting

Forward primer no., sequence

Reverse primer no., sequence

IRAIN JH248, 5'-CGACACATGGTCCAATCACTGTT-3'

IRAIN JH780, 5'-GTTTCCGCAGTAGCCGCTGAT-3'

IRAIN JH249, 5'-AGACTCCCCTAGGACTGCCATCT-3'

IRAIN JH781, 5'-CTGCGGGTCTCCGAAGCTC-3'

B, Reverse transcription-quantitative PCR

Forward primer no., sequence

Reverse primer no., sequence

IRAIN LJ21, 5'-CGATGGATACACGTTCTAATGC-3'

$\beta$-actin J880, 5'-AGATCAAGATCATTGCTCCTCCTGA-3'

IGF1R JH217, 5'-GAAGTCTGGCTCCGGAGGAGGGTC-3'

IRAIN LJ22, 5'-CAAACAATCGGGTAGGATGG-3'

$\beta$-actinJ881,5'-ATACTCCTGCTTGCTGATCCACATC-3'

IGF1R JH2 18, 5'-ATGTGGAGGTAGCCCTCGATCAC-3'

C, DNA methylation analysis

Forward primer no., sequence

Reverse primer no., sequence

IRAIN JH852, 5'-TYGGGGGATGGAGGGGTATTAGGGT-3'

IRAIN JH853, 5'-ACAAACRTCTAAATATCCCCRTAA

AAC-3'

PCR reaction conditions and the electrophoresis method were the same as those used for gDNA PCR products. A bright electrophoresis band with clear edges was observed between 100-200 bp. The target strip was excised and purified according to the experimental method described previously article (10), and sent to the Sangon Biotech Co., Ltd. for gene sequencing.

Gene expression and reverse-transcription quantitative (q)PCR. Total RNA was extracted from frozen tissue samples (laryngeal carcinoma tissues, paired adjacent non-malignant tissues and healthy pharynx tissues) using an AxyPrep Total RNA Extraction kit (Corning, Inc.) according to the manufacturer's protocol. The TransScript ${ }^{\circledR}$ All-in-One First-Strand cDNA Synthesis Super Mix for qPCR kit (Beijing Transgen Biotech Co., Ltd.) was used to synthesize cDNA; for first-strand synthesis, RNA was combined with $5 \mathrm{X}$ TransScript ${ }^{\circledR}$ All-in-One Super Mix, gDNA Remover and RNase-free water and incubated at $42^{\circ} \mathrm{C}$ for $15 \mathrm{~min}$ and then at $85^{\circ} \mathrm{C}$ for $5 \mathrm{sec}$. For qPCR, cDNA samples were amplified using a RocheLightCycler 480II RT system (Roche Diagnostics) and TransStart ${ }^{\circledR}$ Top Green qPCR SuperMix kit (Beijing Transgen Biotech Co., Ltd.). According to manufacturer's protocols, qPCR was performed as follows: Initial denaturation at $94^{\circ} \mathrm{C}$ for $30 \mathrm{sec}$, followed by 40 cycles at $94^{\circ} \mathrm{C}$ for $5 \mathrm{sec}$ and $60^{\circ} \mathrm{C}$ for $30 \mathrm{sec}$. Each sample was analyzed in triplicate and data are presented as mean \pm SD. mRNA expression levels of IRAIN and IGF1R were quantitated via normalizing to $\beta$-actin housekeeping gene (Table II; forward primer, J880 and reverse, J881) according to the method previously described in the present study. The PCR primers used for qPCR were: i) IRAIN, forward LJ21 and reverse LJ22; ii) IGF1R, forward JH217 and reverse JH218; and iii) $\beta$-actin, forward J880 and reverse J881 (Table II). The data was analyzed by using $2^{-\Delta \Delta \mathrm{Cq}}$ method (15).
DNA methylation analysis. gDNA was extracted from tumors using the Animal Tissue Genomic DNA Extraction kit (Beijing Zoman Biotechnology Co., Ltd.). gDNA $(2 \mu \mathrm{g})$ was treated with sodium bisulfite [130 $\mu \mathrm{l}$ CT Conversion reagent of EZ DNA Methylation-Gold kit (Zymo Research Corp.)], and unmethylated cytosine was converted into uracil, while methylated cytosine remained as cytosine. Genomic DNA was used for bisulpite conversion with an EZ DNA Methylation-Gold kit (Zymo Research Corp.). The following steps were performed: $98^{\circ} \mathrm{C}$ for $10 \mathrm{~min}, 64^{\circ} \mathrm{C}$ for $2.5 \mathrm{~h}$ and then $4^{\circ} \mathrm{C}$ storage for upto $20 \mathrm{~h}$ according to the manufacturer's instructions. Then, bisulfite sequencing PCR (BSP) primers were designed, and during PCR amplification all uracil was converted to thymine. PCR products were sequenced to determine if there was methylation of CpG sites using the BSP-direct sequencing method (10). PCR reactions were performed using Kantaq1 DNA polymerase (Ab Peptides, Inc.). BSP was used to analyze DNA methylation status. The PCR conditions were: Initial denaturation at $97^{\circ} \mathrm{C}$ for $10 \mathrm{~min}$, followed by 35 cycles at $96^{\circ} \mathrm{C}$ for $20 \mathrm{sec}$, annealing at $64^{\circ} \mathrm{C}$ for $30 \mathrm{sec}$, extension at $72^{\circ} \mathrm{C}$ for $30 \mathrm{sec}$ and completion of the reaction at $72^{\circ} \mathrm{C}$ for $10 \mathrm{~min}$. The primers used for assessing target fragment DNA methylation were forward JH852 and reverse JH853 (Table II). PCR products were separated via 2\% agarose gel electrophoresis and purified with an Axygen DNA Gel Extraction kit (Axygen; Corning, Inc.). The success rate of sequencing can be improved by cloning PCR products into a vector for sequencing (10). The PCR products were cloned into a pUCm-T vector (Sangon Biotech Co., Ltd.) using a PCR Cloning kit (cat. no. B522213; Sangon Biotech Co., Ltd.) and white colonies grown on IPTG/X-gal indicator plates were selected and the sequenced for analysis of $\mathrm{CpG}$ methylation as previously described $(9,10)$. The status of DNA methylation varies, depending on cell types, microenvironment, gene activity and 
Table III. Expression of 'A' and ' $G$ ' in laryngeal cancer tissue samples and paracanerous tissues.

\begin{tabular}{lcccrr}
\hline Cancer tissue no. & A:G & Ratio & Paracancerous tissue no. & A:G & Ratio \\
\hline CA 1 & $640: 547$ & 1.170 & PC1 & $724: 509$ & 1.422 \\
CA 2 & $313: 405$ & 0.773 & PC2 & $321: 408$ & 0.787 \\
CA 3 & $661: 501$ & 1.320 & PC3 & $648: 536$ & 1.209 \\
CA 4 & $337: 384$ & 0.878 & PC4 & $713: 446$ & 1.599 \\
CA 5 & $724: 544$ & 1.331 & PC5 & $634: 536$ & 1.183 \\
CA 6 & $705: 454$ & 1.553 & PC6 & $667: 539$ & 1.237 \\
CA 7 & $544: 667$ & 0.816 & PC7 & $686: 520$ & 1.320 \\
CA 8 & $689: 522$ & 1.320 & PC8 & $640: 522$ & 1.226 \\
CA 9 & $610: 610$ & 1.000 & PC9 & $683: 544$ & 1.256 \\
CA 10 & $653: 495$ & 1.319 & PC10 11 & $302: 405$ & 0.746 \\
CA 11 & $770: 48$ & 1.598 & PC12 & $599: 476$ & 1.258 \\
CA 12 & $648: 522$ & 1.241 & PC13 & $637: 541$ & 1.177 \\
CA 13 & $898: 495$ & 1.814 & PC14 & $683: 525$ & 1.301 \\
CA 14 & $479: 705$ & 0.679 & PC15 & $672: 571$ & 1.177 \\
CA 15 & $618: 566$ & 1.092 & PC16 & $582: 618$ & 0.942 \\
CA 16 & $552: 501$ & 1.102 & PC17 & $371: 482$ & 1.185 \\
CA 17 & $321: 419$ & 0.766 & & $324: 384$ & 0.844 \\
\hline
\end{tabular}

CA, cancer; AC, adjacent.

Table IV. Expression of ' $A$ ' and ' $G$ ' in healthy pharynx tissues.

\begin{tabular}{lcc}
\hline Healthy pharynx tissue no. & A:G & Ratio \\
\hline N1 & $354: 438$ & 0.808 \\
N2 & $364: 394$ & 0.924 \\
N3 & $373: 413$ & 0.903 \\
N5 & $356: 408$ & 0.873 \\
N6 & $373: 416$ & 0.897 \\
\hline
\end{tabular}

$\mathrm{N}$, healthy.

drug treatment (10). Thus, no known positive controls could be added in the assay for laryngeal cancer tissues.

Statistical analysis. Categorical variables are presented as absolute (n) and relative (\%) frequencies, while continuous variables are presented as medians (min, max). Each sample was analyzed in triplicate and data are presented as mean \pm SD. All data calculations and statistical testing was performed using SPSS version 22.0 statistical software (IBM Corp.). Differences between groups were compared using the Student t-test or the Wilcoxon test and corrected by Bonferroni test, $\mathrm{P}<0.05$ was considered to indicate a statistically significant difference.

\section{Results}

Non-imprinted expression of IRAIN in healthy pharynx tissue, laryngeal carcinoma tissue and paracancerous tissue. To determine whether IRAIN uses a similar epigenetic mechanism to regulate genes locally in laryngeal carcinoma, it was examined if IRAIN IncRNA was mono-allelically expressed in healthy pharynx tissue, laryngeal carcinoma and paracancerous tissue, which are heterozygous for the polymorphic Ndel restriction site. In total, two alleles, termed 'A' allele and ' $\mathrm{G}$ ' allele, were detected in both gDNA and corresponding cDNA samples (Tables III and IV). This indicated that IRAIN lncRNA expression was non-imprinted in healthy pharynx tissue, laryngeal carcinoma tissue and paracancerous tissue.

Then, the allelic expression of IRAIN lncRNA in laryngeal carcinoma tissue and paracancerous tissue was investigated using SNP rs8034564 to distinguish the two parental alleles. As this SNP does not contain a restriction enzyme site, PCR sequencing was used to determine the allelic expression of IRAIN. The gDNAs of laryngeal carcinoma tissues that were heterogeneous for this SNP, including both the 'A' and ' $G$ ' alleles, were selected for analysis. The gDNA PCR products of 32 laryngeal carcinoma tissues and 6 healthy pharyngeal mucosa tissues were sequenced (sent to the Shanghai Sangon Biotech Co., Ltd. for gene sequencing). The SNP site (rs8034564) of 17 laryngeal carcinoma tissues and five healthy pharyngeal mucosa tissues were A/G heterozygous. SNP sequencing was then performed on the cDNA of laryngeal carcinoma tissue and paracancerous tissue of these patients who were heterozygous. However, it was found that the 'A' and ' $G$ ' alleles were detected in all corresponding cDNA samples (Fig. 1). This finding suggested that IRAIN was non-imprinted in laryngeal carcinoma tissue, which was different compared with that in leukemia cells (9) and breast cancer tissue (10).

Allelic expression imbalance and allelic-switch of IRAIN in laryngeal cancer. AEI was used to detect the expression difference of two alleles in the same individual via a heterozygous 
Table V. Association between expression of IRAIN in laryngeal carcinoma and clinical features.

\begin{tabular}{lccc}
\hline Clinical features & Patients $(\mathrm{n}=31)$ & Mean $\pm \mathrm{SD}$ & Shapiro-Wilk value \\
\hline Age, years & 13 & $2.344 \pm 3.681$ & 0.000 \\
$\quad<62$ & 18 & $1.607 \pm 1.707$ & 0.007 \\
$\geq 62$ & & & 0.828 \\
TNM stage & 14 & $1.401 \pm 1.932$ & 0.000 \\
T1-T2 stage & 17 & $1.575 \pm 1.376$ & 0.002 \\
T3-T4 stage & & & 0.000 \\
Histological type & 10 & $1.690 \pm 2.171$ & 0.003 \\
High differentiation & 21 & $1.359 \pm 1.697$ & 0.852 \\
Medium and low differentiation & & & 0.004 \\
Lymph nodes metastasis & 9 & $1.200 \pm 1.519$ & 0.009 \\
$\quad$ Yes & 22 & $1.110 \pm 1.337$ & 0.564 \\
No & & & 0.001 \\
Clinical classification & 15 & $1.514 \pm 1.879$ & 0.000 \\
Glottic carcinoma & 16 & $1.369 \pm 1.358$ & 0.423 \\
Supraglottic carcinoma & & & \\
\hline
\end{tabular}

Analysis of sex was not performed as 28 tissue samples were from males and three were from females, and the disparity would mean that any statistical analysis results would have been unreliable.

SNP site. An allelic expression ratio $(\mathrm{AER})<0.8$ or $>1.2$ are regarded as evidence of AEI $(16,17)$.

The results indicated that the expression of the 'A' allele was favored over the ' $G$ ' allele in laryngeal cancer tissue samples, and their adjacent tissue counterparts (Table III). However, the expression of the ' $G$ ' allele was favored over the 'A' allele in healthy pharynx tissue (Table IV). That suggested IRAIN can cause A:G allelic switch in laryngeal cancer. The A/G value between cancer tissue and healthy pharynx tissue, paracancerous tissue and healthy pharynx tissue were significantly different $(\mathrm{P}=0.009$, $\mathrm{P}=0.033$ respectively), whereas the value between cancer tissue and paracancerous tissue not significantly different (Fig. 2).

Decreased expression of IRAIN in laryngeal cancer types. To evaluate the role of IRAIN in laryngeal cancer, 31 paired laryngeal carcinoma tissues and paracancerous tissue were analyzed for IRAIN expression using RT-qPCR. It was identified that IRAIN expression was significantly decreased in laryngeal cancer tissue ( $\mathrm{P}=0.003$; Fig. 3$)$. Next, the association between IRAIN expression and clinicopathological parameters, including age, histological type, tumor stage, tumor grade and lymph node metastasis, was examined. The analysis indicated that there was no association between IRAIN expression and age, histological type, tumor stage, tumor grade and lymph node metastasis ( $\mathrm{P}>0.05$; Table III).

DNA methylation in the IRAIN promoter. The IRAIN promoter is rich in $\mathrm{CpG}$ dinucleotides, and in peripheral blood leucocytes, the promoter $\mathrm{CpG}$ islands are semi-methylated (9). Compared with peripheral blood leucocytes, Kang et al (10) reported there was aberrant DNA methylation in the IRAIN promoter in breast cancer tissue specimens. The present study analyzed DNA methylation in the IRAIN promoter of laryngeal carcinoma tissue and paracancerous tissue. It was found that the IRAIN promoter was almost completely unmethylated in one paracancerous tissue sample (Fig. 4A). Moreover, the IRAIN promoter was almost completely unmethylated in one laryngeal cancer sample (Fig. 4D). In the other laryngeal cancer samples (Fig. 4B and C), the IRAIN promoter was totally unmethylated, as was seen in the laryngeal carcinoma adjacent tissue. In the laryngeal cancer tissues of three patients (Fig. 4B-D), which contained 13 genes $\mathrm{CpG}$ identified via bisulfite sequencing method, it was found that the laryngeal cancer tissue IRAIN promoter region was completely unmethylated or almost completely unmethylated. Therefore, it was suggested that DNA methylation was not involved in regulation of laryngeal cancer tissues IRAIN genetic imprinting.

\section{Discussion}

lncRNAs are non-protein-coding RNA transcripts $>200$ nucleotides, and are emerging as key regulators of diverse cellular processes (18), such as lncRNA-Xist, lncRNA-HOTAI, IncRNA-H19 and lncRNA-CRNDE. The discovery of lncRNAs as biologically relevant molecules has led to a rethinking of the central dogma of biology and revealed novel cellular and molecular complexities; it is now known that numerous genes can encode both mRNA and lncRNA (19).

Previous studies have reported that lncRNAs serve important functions in the regulation of gene expression and other biological processes, and are dysregulated in tumorigenesis (20). For example, lncRNAs have been identified to exert important roles in tumor recurrence, including $\mathrm{X}$ inactive specific transcript, metastasis associated lung adenocarcinoma transcript 1, HOX transcript antisense RNA, heart and neural crest derivatives expressed 2 and LINC00675. In addition, increased expression of IncRNA H19 imprinted maternally 
A

B

C

GGGACCGCGGCCAAGGGAGGCGGGACCGCGGCCAAGGGAGGCTIGGGACCGCGGCCAAGGGAGGCII

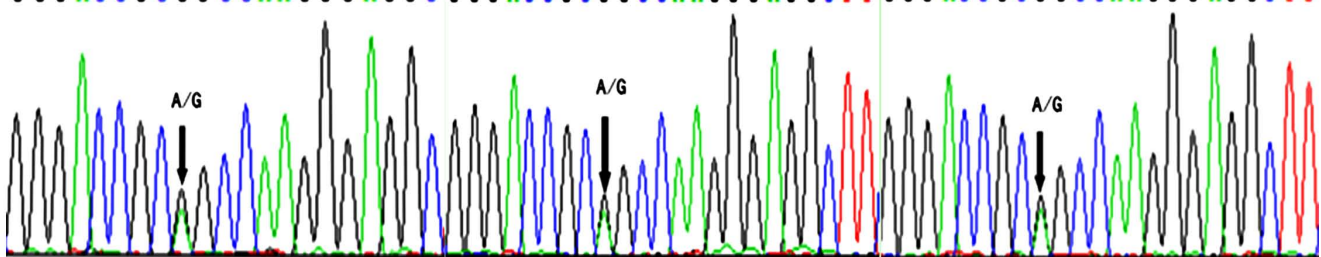

D

E

CGGGACCGCGGCCAAGGGGGGACCGCGGCCAAGGGAGGCI

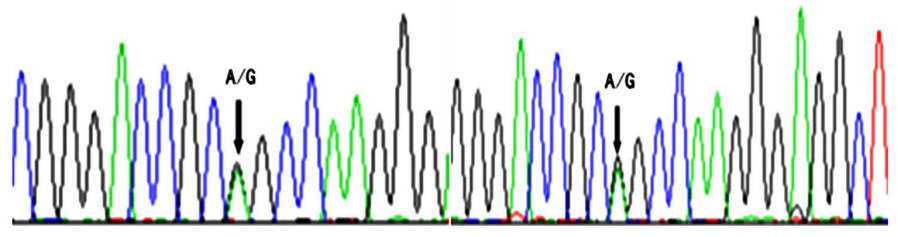

Figure 1. Comparison of IncRNA-IRAIN expression of gDNA and cDNA in healthy pharynx tissue, laryngeal carcinoma tissue and paracancerous tissues. The single 'A' allele and single ' $G$ ' allele of IRAIN IncRNA were detected in samples, it was a heterozygous genomic DNA. The arrow denotes the expression site of A/G. (A) Case one laryngeal carcinoma gDNA (A/G). (B) Case one laryngeal carcinoma cDNA (A/G). (C) Case one laryngeal paracancerous tissue cDNA (A/G). (D) Case two healthy pharynx tissue gDNA (A/G). (E) Case two healthy pharynx tissue cDNA (A/G). gDNA, genomic DNA; cDNA, complementary DNA; lncRNA, long non-coding RNA; IRAIN, IGF1R antisense imprinted non-protein coding RNA.

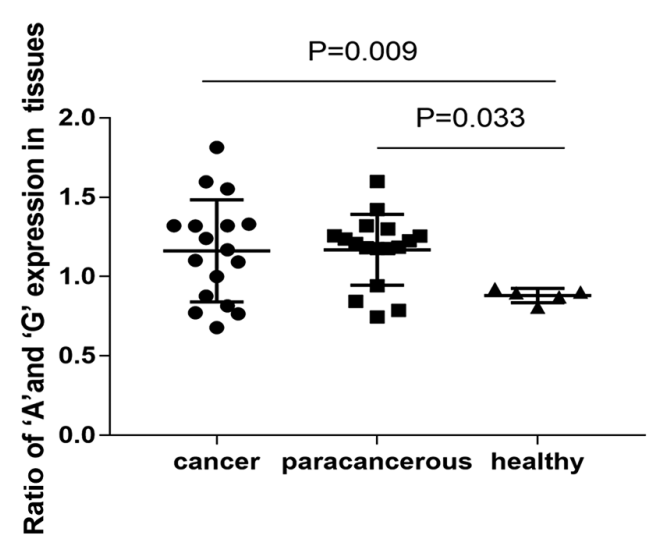

Figure 2. A:G allelic switch between healthy pharynx tissue, cancer tissues and paracancerous tissues. Data are presented as the mean \pm SD. Student t-test with Bonferroni correction was performed to compare statistical differences among treatment groups.

expressed transcript (H19) has been observed in various primary and metastatic tumors, including breast cancer (21), gastric cancer (22), liver cancer (23) and bladder cancer (24). Another previous study has reported that IncRNA H19 promoted laryngeal squamous cell carcinoma progression via microRNA (miR)-148a-3p and DNA methyltransferase 1 (25). It has also been shown that colon cancer-associated transcript-1 (26), taurine upregulated 1 (27) and nuclear paraspeckle assembly transcript 1 (28) are involved in the progression of laryngeal squamous cell cancer, and are associated with TNM stage, tumor size, overall survival and growth regulation of laryngeal squamous cell cancer. Collectively, previous studies have suggested that the identification and investigation of cancer-associated lncRNAs may result in the development of early diagnostic marker and new prognostic biomarkers or treatments for various malignancies, including laryngeal carcinoma.

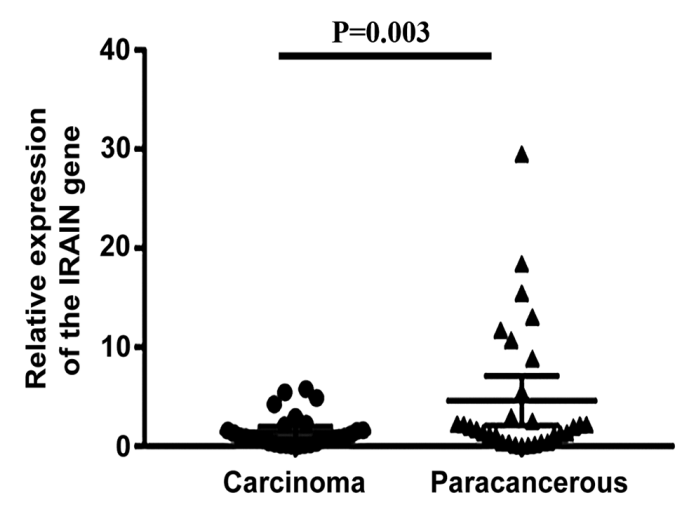

Figure 3. Expression of IRAIN in laryngeal carcinoma and paracancerous tissues, relative expression of the IRAIN gene is $2^{-\Delta \Delta C q}$ value. The Wilcoxon test was used to analyze the difference of pairwise samples. IRAIN, IGF1R antisense imprinted non-protein coding RNA.

Dysregulation of IGF1R has been implicated in the progression of different malignancies, and resistance to treatments, such as breast cancer (10), squamous-cell laryngeal cancer (8) and hematopoietic malignancies (9). A previous study developed and validated a multigene predictor of recurrence in early laryngeal cancer (29); the study identified a panel of genes related to the IGF1R pathway that were able to discriminate patients with a poor and a favorable prognosis $(\mathrm{P}<0.0001$ in the training set; $\mathrm{P}=0.0001$ in the validation set $)$. It has also been shown that IGF1R- $\alpha$ protein upregulation may serve as an independent predictor of recurrence and survival in operable laryngeal cancer (8). Another study suggested that downregulation of miR-375 was one of the molecular mechanisms responsible for the progression of laryngeal carcinoma via targeting IGF1R directly, and affecting its downstream AKT signaling pathways (13). IRAIN is a novel IncRNA within the IGF1R locus that is transcribed in an antisense direction from an intronic promoter, with a full-length transcript of $5.4 \mathrm{~kb}$ (10). IRAIN has been revealed to serve a 


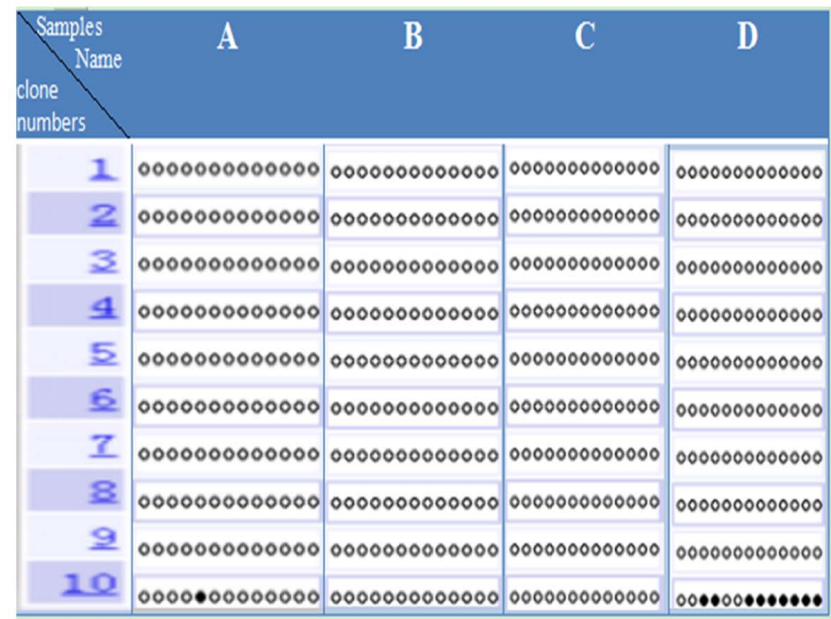

Figure 4. DNA methylation status of the IGF1R/IRAIN gene locus. (A) DNA methylation of the IRAIN promoter in paracancerous tissue. (B-D) DNA methylation status of the IGF1R/IRAIN gene locus of 3 patients with laryngeal carcinoma. Open circles, unmethylated CpGs; solid circles, methylated CpGs; IRAIN, IGF1R antisense imprinted non-protein coding RNA; IGF1R, IRAIN and Insulin-like growth factor 1 receptor.

role in AML (9-12), breast cancer (10), NSCLC (11), renal cell carcinoma (30) and pancreatic cancer (31).

In mice, the gene transcribing IGF2R is associated with a long non-coding (lnc)RNA, antisense of IGF2R non-protein coding RNA (Airn). These transcripts are reciprocally imprinted, with Airn transcribed from the paternal allele only, and the transcription of the antisense lncRNA Airn regulates in cis the allelic expression of the IGF2R coding RNA (32-35). In addition, Sun et al (9) reported that in leukemia cells IRAIN was expressed solely from the paternal allele.

In the present study, it was demonstrated that IRAIN exhibited non-imprinted expression in laryngeal carcinoma tissue, healthy pharynx tissue and paracancerous tissue. However, these results are contrary to those of previous studies examining IRAIN in AML and breast cancer $(9,10)$. These different findings may be because lncRNAs exhibits marked tissue-specific expression patterns, as compared with protein-coding genes, and imprinted genes develop acquired instability $(36,37)$.

If a gene is expressed equally from both alleles (bi-allelically), equivalent levels of each allele of the tracer SNP will be detected in mRNA transcripts (16); this is referred to as 'balanced allelic expression'. When the expression of one allele is altered due to a pathological defect, such as the presence of aberrant promoter methylation or a frameshift mutation that results in nonsense-mediated mRNA decay, the level of one allele will exceed the other in the tracer SNP within the corresponding mRNA; this is generally referred to as 'allelic expression imbalance' (AEI) $(16,17)$. AEI was used to detect the expression difference of two alleles in the same individual using heterozygous SNPs in the current study. SNPs within a gene of interest may be used to distinguish between two genetic alleles, and investigate their features in heterozygous individuals (16). With regards to cancer etiology and development, identification of alleles and the detection of allelic imbalances, such as transcriptional loss from one allele or loss-of-heterozygosity due to deletion of one allele, within a tumor are particularly useful (17). Moreover, it has been reported that an AER $<0.08$ or $>1.2$ is consistent with AEI $(17,38)$. The present results indicated that the IncRNA-IRAIN appeared to favor the ' $A$ ' genotype expression in laryngeal cancer tissue and paracancerous tissue. The expression of the ' $A$ ' allele was greater compared with the ' $\mathrm{G}$ ' allele in $71 \%$ of the 17 heterozygous laryngeal carcinoma tissue specimens examined. The same result was found in the examination of paracancerous tissue specimens (cA $>\mathrm{G}$; rs8034564). However, the expression of the ' $G$ ' allele was favored over the ' $A$ ' allele in healthy pharynx tissue specimens $(c A<G$, rs8034564). These results demonstrated that the expression of IRAIN IncRNA switches to the alternate parental allele in laryngeal carcinoma tissue and paracancerous tissue. However, the mechanisms underlying the AEI and allele-switch in laryngeal carcinoma remains unknown. It was hypothesized that the mechanism may be associated with the two characteristics of lncRNA: Inc RNA expression possesses cell or tissue type specificity and lncRNA is less conserved compared with mRNA. Moreover, whether the AEI and aberrant allelic switch affects the activity of the IGF1R signaling pathway in laryngeal carcinoma is yet to be elucidated, and thus requires further research in the future.

The current results indicated that IRAIN expression was decreased in laryngeal carcinoma tissue, which is consistent with the findings of previous studies of AML $(9,12)$, breast cancer (10) and renal cell carcinoma (30). However, the results are contrary to the findings of a study in NSCLC (11); this may be due to the fact that lncRNAs have tissue-specific expression patterns, which is different compared with the expression patterns of protein-coding genes $(36,37)$. The present study also identified no associations between IRAIN expression and clinical characteristics, such as age, histological type, tumor stage and grade and lymph node metastasis. According these results, it was suggested that IRAIN may exert an important role in laryngeal carcinoma development and progression. Furthermore, IRAIN may serve as a novel tumor suppressing lncRNA and as an early diagnostic marker in laryngeal cancer.

Mammals are diploid organisms whose cells possess two matched sets of chromosomes, one inherited from the mother and one from the father $(10,39)$. Thus, mammals have two copies of every gene. Normally, both the maternal and paternal copy of each gene have the same potential to be active in any cell (39). Genomic imprinting is an epigenetic mechanism that changes this potential by restricting the expression of a gene to one of the two parental chromosomes $(9,10,39)$. DNA methylation could potentially perform two different functions in genomic imprinting (39); for example, it could act as the imprinting mark via being acquired de novo only by the chromosomes in one gamete (39). Moreover, DNA methylation could serve to silence one of the parental alleles, as it is associated with gene repression (40). In the current study, it was demonstrated that the IRAIN promoter was unmethylated in one paracancerous tissue specimen and in three laryngeal carcinoma tissue specimens. Thus, the results indicated that DNA methylation was not involved in gene imprinting expression in the promoter region of IRAIN; 
however, these findings may provide a reference for further studies regarding lncRNA and the pathogenesis of laryngeal cancer.

In conclusion, the present results suggested that IRAIN expression was significantly decreased in laryngeal carcinoma tissue, and that IRAIN undergoes AEI and aberrant allelic switching in laryngeal cancer. However, the present study had several limitations. Firstly, the small sample size of laryngeal carcinoma tissues and paracanerous tissues due to difficulty of collection. Secondly, the number of individuals from whom healthy pharynx tissue was obtained was lower compared to the patients from whom laryngeal carcinoma and paracancerous tissues were obtained. Thirdly, in the present study, the number of female patients were lower compared to male patients leading to a sex bias. Additional studies are required to further verify the current findings and investigate the unanswered questions, including whether AEI and the aberrant allele-switch of IRAIN lncRNA serve an important role in the development of laryngeal cancer? If IRAIN lncRNA could be used to predict the effectiveness of IGF1R targeted therapies? Or whether IRAIN IncRNA can be used to as a potential therapeutic target in laryngeal carcinoma treatment? Furthermore, it would be also be of interest to investigate whether AEI and the aberrant allelic switching of IRAIN lncRNA can be used to assess laryngeal cancer risk.

\section{Acknowledgements}

Not applicable.

\section{Funding}

This study was funded by the Natural Science Funds of Jilin Province, China (grant no. 20160101055JC).

\section{Availability of data and materials}

The datasets used in the present study are available from the corresponding author upon reasonable request.

\section{Authors' contributions}

JW and MZ conceived and designed the research methods, as well as drafted and revised the manuscript. JW and YM performed the experiments. JW, DL and YG analyzed the data and prepared the figures. JW assisted with the analysis of the data and interpreted the results of the experiments. All authors approved the final version of the manuscript, as well as agree to be accountable for all aspects of the research and for ensuring the accuracy and integrity of the study.

\section{Ethics approval and consent to participate}

The experimental protocol was approved by the Ethics Committee of the Second Hospital of Jilin University, and written informed consent was obtained from all patients.

\section{Patient consent for publication}

Not applicable.

\section{Competing interests}

The authors declare that they have no competing interests.

\section{References}

1. Haapaniemi A, Koivunen P, Saarilahti K, Kinnunen I, Laranne J, Aaltonen LM, Närkiö M, Lindholm P, Grénman R, Mäkitie A, et al: Laryngeal cancer in Finland: A 5-year follow-up study of 366 patients. Head Neck 38: 36-43, 2016.

2. Steuer CE, El-Deiry M, Parks JR, Higgins KA and Saba NF: An update on larynx cancer. CA Cancer J Clin 67: 31-50, 2017.

3. Gong H, Shi Y, Xiao X, Cao P, Wu C, Tao L, Hou D, Wang Y and Zhou L: Alterations of microbiota structure in the larynx relevant to laryngeal carcinoma. Sci Rep 7: 5507, 2017.

4. Rudolph E, Dyckhoff G, Becher H, Dietz A and Ramroth H: Effects of tumour stage, comorbidity and therapy on survival of laryngeal cancer patients: A systematic review and a meta-analysis. Eur Arch Otorhinolaryngol 268: 165-179, 2011.

5. Bach LA: IGF-binding proteins. J Mol Endocrinol 61: T11-T28, 2018.

6. Jal L, Ram M, Akl R, van der Zee AGJ and de Jong S: IGF system targeted therapy: Therapeutic opportunities for ovarian cancer. Cancer Treat Rev 60: 90-99, 2017.

7. Winder T, Zhang W, Yang D, Ning Y, Bohanes P, Gerger A, Wilson PM, Pohl A, Mauro DJ, Langer C, et al: Germline polymorphisms in genes involved in the IGF1 pathway predict efficacy of cetuximab in wild-type KRAS mCRC patients. Clin Cancer Res 16: 5591-5602, 2010.

8. Mountzios G, Kostopoulos I, Kotoula V, Sfakianaki I, Fountzilas E, Markou K, Karasmanis I, Leva S, Angouridakis N, Vlachtsis K, et al: Insulin-like growth factor 1 receptor (IGF1R) expression and survival in operable squamous-cell laryngeal cancer. PLoS One 8: e54048, 2013.

9. Sun J, Li W, Sun Y, Yu D, Wen X, Wang H, Cui J, Wang G, Hoffman AR and Hu JF: A novel antisense long noncoding RNA within the IGF1R gene locus is imprinted in hematopoietic malignancies. Nucleic Acids Res 42: 9588-9601, 2014.

10. Kang L, Sun J, Wen X, Cui J, Wang G, Hoffman AR, Hu JF and Li W: Aberrant allele-switch imprinting of a novel IGF1R intragenic antisense non-coding RNA in breast cancers. Eur J Cancer 51: 260-270, 2015.

11. Feng J, Sun Y, Zhang EB, Lu XY, Jin SD and Guo RH: A novel long noncoding RNA IRAIN regulates cell proliferation in non small cell lung cancer. Int J Clin Exp Pathol 8: 12268-12275, 2015.

12. Pashaiefar $\mathbf{H}$, Izadifard $\mathbf{M}$, Yaghmaie $M$, Montazeri $M$, Gheisari E, Ahmadvand M, Momeny M, Ghaffari SH, Kasaeian A, Alimoghaddam K and Ghavamzadeh A: Low expression of long noncoding RNA IRAIN is associated with poor prognosis in Non-M3 acute myeloid leukemia patients. Genet Test Mol Biomarkers 22: 288-294, 2018.

13. Luo J, Wu J, Li Z, Qin H, Wang B, Wong TS, Yang W, Fu QL and Lei W: miR-375 suppresses IGF1R expression and contributes to inhibition of cell progression in laryngeal squamous cell carcinoma. Biomed Res Int 2014: 374598, 2014.

14. National Comprehensive Cancer Network (NCCN): NCCN Clinical Practice Guidelines in Oncology-Head and Neck Cancers. Version 1.2018. NCCN, Plymouth Meeting, PA, 2018. https://www.nccn.org/professionals/physician_gls/default.aspx.

15. Livak KJ and Schmittgen TD: Analysis of relative gene expression data using real-time quantitative PCR and the 2(-Delta Delta C(T)) method. Methods 25: 402-408, 2001.

16. KwokCT and Hitchins MP: Allele quantification Pyrosequencing ${ }^{\circledR}$ at designated SNP sites to detect allelic expression imbalance and Loss-of-Heterozygosity. Methods Mol Biol 1315: 153-171, 2015.

17. Wang ZM, Wang DZ and Shi JX: Detection and application of allelic expression imbalance. Zhonghua Yu Fang Yi Xue Za Zhi 45: 9-11, 2011 (In Chinese).

18. Sun $M$ and Kraus WL: From discovery to function: The expanding roles of long noncoding RNAs in physiology and disease. Endocr Rev 36: 25-64, 2015.

19. Grelet S, Link LA, Howley B, Obellianne C, Palanisamy V, Gangaraju VK, Diehl JA and Howe PH: A regulated PNUTS mRNA to lncRNA splice switch mediates EMT and tumour progression. Nat Cell Biol 19: 1105-1115, 2017.

20. Ren S, Wang F, Shen J, Sun Y, Xu W, Lu J, Wei M, Xu C, Wu C, Zhang Z, et al: Long non-coding RNA metastasis associated in lung adenocarcinoma transcript 1 derived miniRNA as a novel plasma-based biomarker for diagnosing prostate cancer. Eur J Cancer 49: 2949-2959, 2013. 
21. Collette J, Le Bourhis X and Adriaenssens E: Regulation of human breast cancer by the long Non-coding RNA H19. Int J Mol Sci 18: 2319, 2017.

22. Li H, Yu B, Li J, Su L, Yan M, Zhu Z and Liu B: Overexpression of lncRNA H19 enhances carcinogenesis and metastasis of gastric cancer. Oncotarget 5: 2318-2329, 2014.

23. Conigliaro A, Costa V, Lo Dico A, Saieva L, Buccheri S, Dieli F, Manno M, Raccosta S, Mancone C, Tripodi M, et al: CD90+ liver cancer cells modulate endothelial cell phenotype through the release of exosomes containing H19 lncRNA. Mol Cancer 14: $155,2015$.

24. Lv M, Zhong Z, Huang M, Tian Q, Jiang R and Chen J: lncRNA H19 regulates epithelial-mesenchymal transition and metastasis of bladder cancer by miR-29b-3p as competing endogenous RNA Biochim Biophys Acta Mol Cell Res 1864: 1887-1899, 2017.

25. Wu T, Qu L, He G, Tian L, Li L, Zhou H, Jin Q, Ren J, Wang Y, Wang J, et al: Regulation of laryngeal squamous cell cancer progression by the lncRNA H19/miR-148a-3p/DNMT1 axis Oncotarget 7: 11553-11566, 2016.

26. Zhang Y and Hu H: Long non-coding RNA CCAT1/miR-218/ZFX axis modulates the progression of laryngeal squamous cell cancer. Tumour Biol 39: 1010428317699417, 2017.

27. Zhang Z, Wang X, Cao S, Han X, Wang Z, Zhao X, Liu X, Li G, Pan X and Lei D: The long noncoding RNA TUG1 promotes laryngeal cancer proliferation and migration. Cell Physiol Biochem 49: 2511-2520, 2018.

28. Wang $\mathrm{P}, \mathrm{Wu} \mathrm{T}$, Zhou H, Jin Q, He G, Yu H, Xuan L, Wang X, Tian L, Sun Y, et al: Long noncoding RNA NEAT1 promotes laryngeal squamous cell cancer through regulating miR-107/CDK6 pathway. J Exp Clin Cancer Res 35: 22, 2016.

29. Fountzilas E, Kotoula V, Angouridakis N, Karasmanis I, Wirtz RM, Eleftheraki AG, Veltrup E, Markou K, Nikolaou A, Pectasides D and Fountzilas G: Identification and validation of a multigene predictor of recurrence in primary laryngeal cancer. PLoS One 8: e70429, 2013.

30. Wang Z, Liu Q, Tieqiang L, Xiaodong L, Guangwei Z, Yang L, Hao $Z$ and Chaoyang $Z$ : Abnormal expressed long non-coding RNA IRAIN inhibits tumor progression in human renal cell carcinoma cells. Open Life Sci 11: 200-205, 2016.
31. Lian Y, Wang J, Feng J, Ding J, Ma Z, Li J, Peng P, De W and Wang K: Long non-coding RNA IRAIN suppresses apoptosis and promotes proliferation by binding to LSD1 and EZH2 in pancreatic cancer. Tumour Biol 37: 14929-14937, 2016.

32. Hu JF, Balaguru KA, Ivaturi RD, Oruganti H, Li T, Nguyen BT, $\mathrm{Vu}$ TH and Hoffman AR: Lack of reciprocal genomic imprinting of sense and antisense RNA of mouse insulin-like growth factor II receptor in the central nervous system. Biochem Biophys Res Commun 257: 604-608, 1999.

33. Hu JF, Pham J, Dey I, Li T, Vu TH and Hoffman AR: Allele-specific histone acetylation accompanies genomic imprinting of the insulin-like growth factor II receptor gene. Endocrinology 141: 4428-4435, 2000.

34. Stöger R, Kubicka P, Liu CG, Kafri T, Razin A, Cedar H and Barlow DP: Maternal-specific methylation of the imprinted mouse Igf $2 \mathrm{r}$ locus identifies the expressed locus as carrying the imprinting signal. Cell 73: 61-71, 1993.

35. Wutz A, Smrzka OW, Schweifer N, Schellander K, Wagner EF and Barlow DP: Imprinted expression of the Igf2r gene depends on an intronic $\mathrm{CpG}$ island. Nature 389: 745-749, 1997.

36. Cabili MN, Trapnell C, Goff L, Koziol M, Tazon-Vega B, Regev A and Rinn JL: Integrative annotation of human large intergenic noncoding RNAs reveals global properties and specific subclasses. Genes Dev 25: 1915-1927, 2011.

37. Derrien T, Johnson R, Bussotti G, Tanzer A, Djebali S, Tilgner H, Guernec G, Martin D, Merkel A, Knowles DG, et al: The GENCODE v7 catalog of human long noncoding RNAs: Analysis of their gene structure, evolution, and expression. Genome Res 22: 1775-1789, 2012.

38. Yan H, Yuan W, Velculescu VE, Vogelstein B and Kinzler KW: Allelic variation in human gene expression. Science 297: 1143, 2002.

39. Barlow DP and Bartolomei MS: Genomic imprinting in mammals. Cold Spring Harb Perspect Biol 6: a018382, 2014.

40. Li E and Zhang Y: DNA methylation in mammals. Cold Spring Harb Perspect Biol 6: a019133, 2014.

This work is licensed under a Creative Commons Attribution-NonCommercial-NoDerivatives 4.0 International (CC BY-NC-ND 4.0) License. 